\title{
Chemiresistive Properties of Imprinted Fluorinated Graphene Films
}

\author{
Vitalii I. Sysoev ${ }^{1, *}\left(\mathbb{C}\right.$, Mikhail O. Bulavskiy ${ }^{1,2}$, Dmitry V. Pinakov ${ }^{1}\left(\mathbb{D}\right.$, Galina N. Chekhova ${ }^{1}$, \\ Igor P. Asanov ${ }^{1} \mathbb{D}$, Pavel N. Gevko ${ }^{1}$, Lyubov G. Bulusheva ${ }^{1}\left(\mathbb{D}\right.$ and Alexander V. Okotrub ${ }^{1, *}$ \\ 1 Nikolaev Institute of Inorganic Chemistry SB RAS, 3 Acad. Lavrentiev Ave., 630090 Novosibirsk, Russia; \\ mikhail@bulavsky.pp.ru (M.O.B.); pinakov@niic.nsc.ru (D.V.P.); chekhova@niic.nsc.ru (G.N.C.); \\ asan@niic.nsc.ru (I.P.A.); paul@niic.nsc.ru (P.N.G.); bul@niic.nsc.ru (L.G.B.) \\ 2 Faculty of Natural Sciences, Novosibirsk State University, 2 Pirogova Str., 630090 Novosibirsk, Russia \\ * Correspondence: sysoev@niic.nsc.ru (V.I.S.); spectrum@niic.nsc.ru (A.V.O.); Tel.: +7-383-3305352 (A.V.O.)
}

Received: 16 July 2020; Accepted: 7 August 2020; Published: 11 August 2020

\begin{abstract}
The electrical conductivity of graphene materials is strongly sensitive to the surface adsorbates, which makes them an excellent platform for the development of gas sensor devices. Functionalization of the surface of graphene opens up the possibility of adjusting the sensor to a target molecule. Here, we investigated the sensor properties of fluorinated graphene films towards exposure to low concentrations of nitrogen dioxide $\mathrm{NO}_{2}$. The films were produced by liquid-phase exfoliation of fluorinated graphite samples with a composition of $\mathrm{CF}_{0.08}, \mathrm{CF}_{0.23}$, and $\mathrm{CF}_{0.33}$. Fluorination of graphite using a $\mathrm{BrF}_{3} / \mathrm{Br}_{2}$ mixture at room temperature resulted in the covalent attachment of fluorine to basal carbon atoms, which was confirmed by X-ray photoelectron and Raman spectroscopies. Depending on the fluorination degree, the graphite powders had a different dispersion ability in toluene, which affected an average lateral size and thickness of the flakes. The films obtained from fluorinated graphite $\mathrm{CF}_{0.33}$ showed the highest relative response ca. $43 \%$ towards $100 \mathrm{ppm} \mathrm{NO} \mathrm{NO}_{2}$ and the best recovery ca. $37 \%$ at room temperature.
\end{abstract}

Keywords: fluorinated graphene; resistivity; nitrogen dioxide; adsorption; gas sensor

\section{Introduction}

Graphene materials are widely examined as sensitive layers of the resistive gas sensors owing to a strong dependence of the electrical properties on the surrounding medium [1]. It has been demonstrated that graphene-based materials are able to detect various electron-donor $\left(\mathrm{NH}_{3}[2], \mathrm{H}_{2} \mathrm{~S}\right.$ [3], $\left.\mathrm{CO}_{2}[4]\right)$ and electron-acceptor ( $\mathrm{NO}, \mathrm{NO}_{2}[5,6]$, organic nitro-compounds [7]) gases or vapors. Most of the studies are conducted with nitrogen dioxide $\mathrm{NO}_{2}$, as it is a convenient model system. In addition, detection of nitrogen oxides gases is significant in the protection of environmental and human health and the detection of explosives [8,9]. The main disadvantages of graphene-based sensors are slow response and poor recovery that makes them useless for real time sensing at room temperature. The reasons for long response and recovery time are slow adsorption kinetics of molecules and presence of high-energy sites, such as defects and oxygen containing groups [10], which catch adsorbate thus making the sensor recovery difficult. On the other hand, structural defects, edge states, dopants, and functional groups play an important role in the electrical response of graphene [6,11-14]. The controlled introduction of a certain type of defect and functional group enhances the performance of a graphene-based sensor by increasing the binding energy of adsorbate and charge transfer in the reactive sites. Functionalization and doping of graphene include noncovalent modification via $\pi-\pi$ stacking, covalent attachment to the unsaturated double bonds, intercalation [15], and insertion of foreign elements in the graphene lattice [16]. The direct grow of functionalized or doped material is a challenging task, and it is limited 
by precursor selection. The post-synthesis functionalization of graphene prepared using the chemical vapor deposition (CVD) method always requires involvement of complicated techniques, such as ion bombardment, plasma treatment, etc. The use of graphite and graphite derivatives is a more reasonable route for preparation of functionalized graphene.

Halogenation is a promising way to obtained graphene derivatives possessing an energy gap, unique two-dimensional structure, and uniform distribution of functional groups on the surface [17]. As compared to bulky chlorine [18] and bromine [19] atoms, fluorine can attach to every carbon atom up to a fully fluorinated graphene (FG) layer [20]. A strong covalent bonding between carbon and fluorine distorts an initially planar graphene sheet [21] thus creating the active sites for the adsorbate. The amount, distribution, and character of C-F bonds strongly affect the chemical, electrochemical, electrical, electronic, optical, and magnetic properties as well as stability and hydrophobicity of FG [22]. There are different ways of fluorination, such as a treatment in hydrofluoric acid solution [23], hydrothermal methods [24], photonic [25], plasma [26], and by using the gaseous fluorinating agents [22,27]. These methods differ by the efficiency, resultant fluorine pattern [28], and structure of obtained graphite or graphene derivatives. Moreover, single- or few-layered FG can be obtained using liquid phase exfoliation of fluorinated graphite in an organic solvent. The deep understanding of the $\mathrm{CF}_{\mathrm{x}}$ structure is fundamental to control the physicochemical properties of these materials by changing the number of layers, size, and surface chemistry, which determine an application field of the fluorinated graphene.

Herein, we report the preparation of FG films from fluorinated graphites with a composition of $\mathrm{CF}_{0.08}, \mathrm{CF}_{0.23}$, and $\mathrm{CF}_{0.33}$ using a sonication of powders in toluene followed by a vacuum filtration. The films deposited on membranes can be transferred on various substrates, including a flexible polymer substrate by simple imprinted technique. The structure and composition of the FG films were studied by scanning electron microscopy (SEM), energy dispersive X-ray spectroscopy (EDS), atomic force microscopy (AFM), and Raman scattering. We showed that both the fluorination degree and the particle size affect the sensor properties of the films toward gaseous $\mathrm{NO}_{2}$. The functionalization modifies the electronic state of graphene, improving response and recovery of the sensor. Better exfoliation ability of highly fluorinated graphite provides a greater increase in the sensor response as compared to the lower fluorinated films.

\section{Materials and Methods}

Natural graphite, purified from impurities, was fluorinated in Teflon reactors using vapors of $\mathrm{BrF}_{3}$ and $\mathrm{Br}_{2}$ at room temperature. The fluorine content in the product was tuned by changing the concentration $(\mathrm{C})$ of $\mathrm{BrF}_{3}$ diluted by $\mathrm{Br}_{2}$ and the reaction time $(\mathrm{t})$. The details of the synthesis procedure are described elsewhere $[29,30]$. Three samples with different fluorine content were used in this study. The composition of the fluorinated layers determined from elemental analysis was a $\mathrm{CF}_{0.33}$ $\left(\mathrm{C}\left(\mathrm{BrF}_{3}\right)=10\right.$ wt. $\%, \mathrm{t}=30$ days $), \mathrm{CF}_{0.23}\left(\mathrm{C}\left(\mathrm{BrF}_{3}\right)=4.3 \mathrm{wt} . \%, \mathrm{t}=90\right.$ days $)$, and $\mathrm{CF}_{0.08}\left(\mathrm{C}\left(\mathrm{BrF}_{3}\right)=2.1 \%\right.$, $\mathrm{t}=150$ days. Electronic state of the elements in the samples was revealed by $\mathrm{X}$-ray photoelectron spectroscopy (XPS) on a Phoibos 150 Specs spectrometer using monochromatic Al K $\alpha$ (1486.6 eV) radiation. The surface composition was determined from the survey XPS spectra; the high-resolution spectra were fitted using Doniac-Sunjic and Gaussian-Lorentzian peak profiles after subtraction of Shirley-type background.

A fluorinated graphite powder (ca. $1 \mathrm{mg}$ ) mixed with toluene $(10 \mu \mathrm{L})$ was grinded in an agate mortar and then bath-sonicated in toluene $(10 \mathrm{~mL})$ for $30 \mathrm{~min}$. Non-exfoliated particles were removed by sedimentation of dispersion for $1 \mathrm{~h} .9 \mathrm{~mL}$ of the obtained stable dispersion of FG was filtered under vacuum through a nitrate cellulose $(\mathrm{CN})$ membrane with a diameter of $47 \mathrm{~mm}$ and a pore size of $0.45 \mu \mathrm{m}$. The obtained films were designated as FG-s. To increase the degree of exfoliation of fluorinated graphite, the grinded powder was sonicated in $40 \mathrm{~mL}$ of toluene for $30 \mathrm{~min}$. After sedimentation for $1 \mathrm{~h}$, the dispersion was sonicated at $78 \mathrm{~W}$ for $1 \mathrm{~h}$ and centrifuged at $1000 \mathrm{rpm}$ for $15 \mathrm{~min}$ to separate the 
fine particles of FG. The supernatant was additionally sonicated at $78 \mathrm{~W}$ for $15 \mathrm{~min}$ and $35 \mathrm{~mL}$ of the dispersion was filtered to obtain the films denoted FG-c.

FG-s and FG-c films were imprinted to a $\mathrm{SiO}_{2} / \mathrm{Si}$, glass or polyethylene terephthalate (PET) substrate using hot pressing. The $\mathrm{CN}$ membrane with a deposited FG film was cut into pieces of $3 \times 5 \mathrm{~mm}^{2}$, and a piece was put on a substrate with a size of $6 \times 5 \mathrm{~mm}^{2}$ by the side with the film. The substrate was heated to $120^{\circ} \mathrm{C}$, and the load of $1 \mathrm{~kg} / \mathrm{cm}^{2}$ was applied to the assembly for $15 \mathrm{~min}$. The CN membrane was mechanically detached from the FG film, and membrane residues were removed by acetone. To increase the adhesion with silicon or glass substrate, the films were wetted by distilled water. Finally, electrodes were deposited by silver conductive paint (RS Components Ltd, Corby, UK) on two opposite sides of the film that gave a sensitive layer with a size of ca. $3 \times 3 \mathrm{~mm}^{2}$. The samples were annealed at $80^{\circ} \mathrm{C}$, as long as the conductivity did not stop changing.

The sensor test was performed in the experimental gas system described in our previous work [31]. Electrical characterization of the films was carried out using a two-probe technique under atmospheric pressure. The current was recorded using a Keithley 6485 picoammeter at a constant voltage in the range from 0.1 to $1 \mathrm{~V}$, depending on film conductivity. The standard cycle of the sensor test consisted of 3 min of exposure to gas mixture (100 ppm $\mathrm{NO}_{2}$ in $\mathrm{Ar}$ ) and $12 \mathrm{~min}$ of recovery in pure Ar. The relative response and recovery were estimated using Equations (1) and (2):

$$
\begin{gathered}
\text { Relative response }(\%)=\frac{I_{g}-I_{0}}{I_{0}} \times 100 \% \\
\text { Recovery }(\%)=\frac{I_{g}-I_{a}}{I_{g}-I_{0}} \times 100 \%
\end{gathered}
$$

where $I_{o}$ and $I_{g}$ are the currents before and after exposure to $\mathrm{NO}_{2}$ and $I_{a}$ is the current after sensor recovery by pure argon.

The characterization was made for FG films on silicon substrates. Raman spectra were recorded using an excitation from an $\mathrm{Ar}^{+}$laser at $514 \mathrm{~nm}$ on a LabRAM HR Evolution (Horiba, Kyoto, Japan) spectrometer. Optical absorbance measurements were performed using an Optizen $220 \mathrm{UV}$ spectrometer (KLAB, Daejeon, Korea). Elemental analysis of the FG films was carried out by EDS on a Bruker QUANTAX spectrometer with an XFlash $6 \mid 60$ detector. Morphology, thickness, and particle sizes of FG films were characterized using a set of microscopic methods, namely optical microscopy on a BX 51TRF microscope (Olympus Corporation, Tokyo, Japan), SEM on a JEOL JSM-6700F microscope (Tokyo, Japan), and AFM on a Solver Pro (NT-MDT) microscope (Moscow, Russia). The AFM measurements were performed in tapping mode using cantilevers NSG10 (NT-MDT) with a tip curvature radius of $6 \mathrm{~nm}$ and an average value of the force constant of $11.8 \mathrm{~N} / \mathrm{m}$.

\section{Results}

\subsection{Materials Characterization}

The content of fluorine in the surface of the fluorinated graphites determined from the XPS survey spectra was ca. 7 at.\% in $\mathrm{CF}_{0.08}$ sample, ca. 19 at.\% in $\mathrm{CF}_{0.23}$ sample, and ca. 25 at.\% in $\mathrm{CF}_{0.33}$ sample. Lower values given by XPS are due to partial hydrolysis of the sample surface by $\mathrm{H}_{2} \mathrm{O}$ present in laboratory air [32].

The XPS C 1s spectra of the samples are compared in Figure 1a. Each spectrum exhibits a set of the components corresponding to (1) $\mathrm{sp}^{2}$-hybridized carbon (ca. $284.5 \mathrm{eV}$ ), (2) carbon atoms located at $\mathrm{C}-\mathrm{F}$ bonds $\left(\mathrm{C}^{*}-\mathrm{C}(\mathrm{F})\right)(\mathrm{ca} .285 .3 \mathrm{eV}),(3) \mathrm{sp}^{3}$ carbon atoms covalently bonded with fluorine atoms $\left(C^{*}-\mathrm{F}\right)(\mathrm{ca} .288 .0 \mathrm{eV})$, and $(4-6)-\mathrm{CF}_{\mathrm{x}}(\mathrm{x}=1-3)$ groups (ca. $\left.289.7-292.0 \mathrm{eV}\right)$ located at crystallite boundaries [33-36]. A broad high-energy component is assigned to $\pi \rightarrow \pi^{*}$ electron excitations. The contribution of $\mathrm{Csp}^{2}$ component to the $\mathrm{C} 1 \mathrm{~s}$ spectrum decreases in the set $\mathrm{CF}_{0.08}>\mathrm{CF}_{0.23}>\mathrm{CF}_{0.33}$, and this indicates a reduction of the average size of graphene-like areas remaining in the layers after the fluorination procedure. A comparable integral intensity of the components $C^{*}-F$ and $C^{*}-C(F)$ 
means the formation of chains from fluorine atoms [37]. The co-existence of patterns from alternating CF chains and bare carbon chains, and graphene islands in the fluorinated layers is typical for the sample obtained by room temperature fluorination of graphite [33].
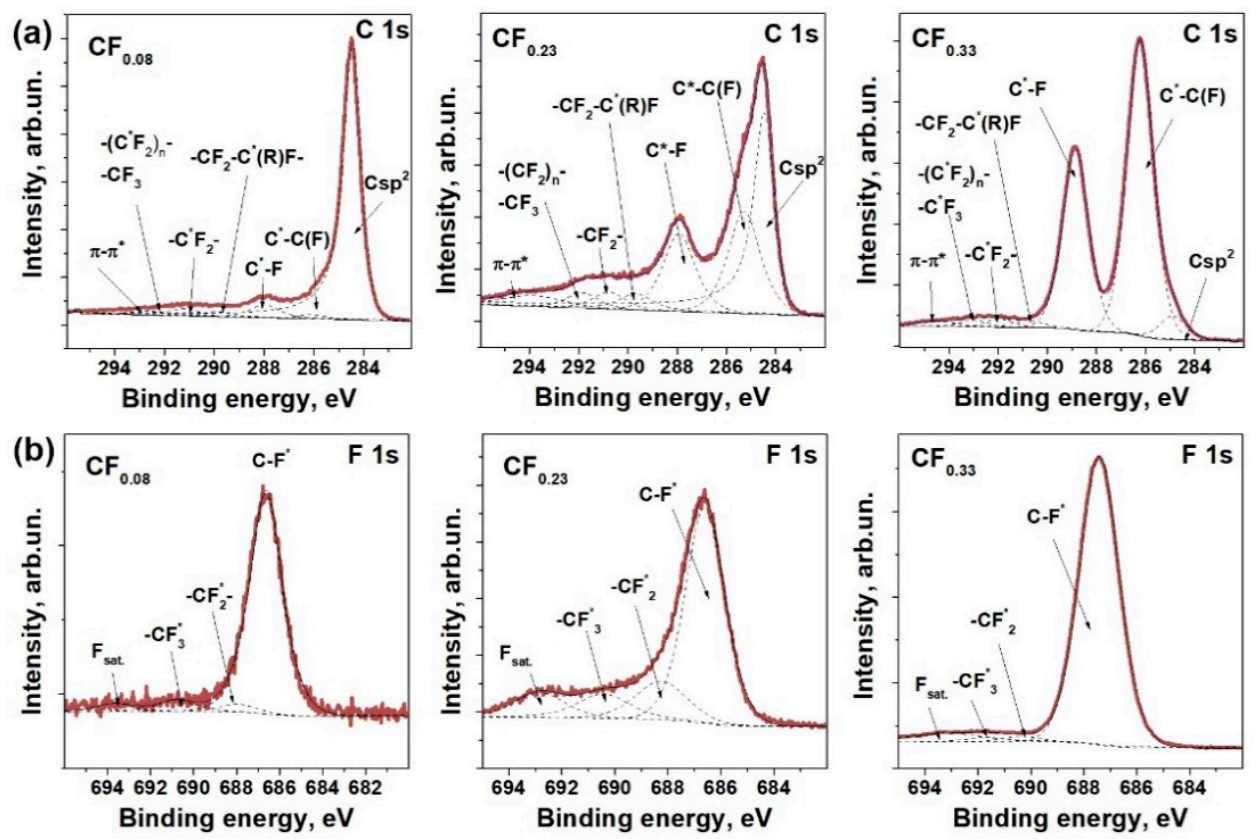

Figure 1. XPS C 1s (a) and F 1s (b) spectra of fluorinated graphites with the composition of layers of $\mathrm{CF}_{0.08}, \mathrm{CF}_{0.23}$, and $\mathrm{CF}_{0.33}$.

The XPS F 1s spectra of all the studied fluorinated graphites exhibited a dominant peak at ca. $687.4 \mathrm{eV}$ (Figure 1b), attributing to weakened covalent C-F bonding [38]. A broad component around ca. $693.0 \mathrm{eV}$ corresponds to the loss-energy satellite. The components between the main peak and the satellite are assigned to $-\mathrm{CF}_{\mathrm{x}}$ groups located at the edges of graphite crystallites [35]. The fraction of these groups is largest $(\sim 40 \%)$ in the $\mathrm{CF}_{0.23}$ sample, as both $\mathrm{F} 1 \mathrm{~s}$ and $\mathrm{C} 1 \mathrm{~s}$ spectra are revealed. It is likely that the holding of graphite in the vapors of highly reactive $\mathrm{BrF}_{3}$ for several months causes etching of graphene layers at defects and grain boundaries accompanying by the formation of $-\mathrm{CF}_{\mathrm{x}}$ groups. Stronger etching observed for $\mathrm{CF}_{0.23}$ can be related to the higher concentration of $\mathrm{BrF}_{3}(4.3 \mathrm{wt}$. \%) in the reaction mixture as compared to that used for the synthesis of $\mathrm{CF}_{0.08}$.

The method used here for the fluorination of graphite leads to the covalent attachment of fluorine atoms to both sides of the graphene planes [32]. As a result, the interlayer distance in fluorinated graphites increases, while the van der Waals forces between the layers decrease as compared to graphite. Weakening of the interaction between the layers allows easy exfoliation of fluorinated graphites in liquid media $[39,40]$. We tried tetrahydrofuran, chloroform, acetonitrile, isopropanol, and toluene. Freshly prepared dispersions of the fluorinated graphites have a yellowish color. This color changed under sonication of the dispersions in acetonitrile and tetrahydrofuran due to partial reduction of fluorinated graphene layers. An ultrasound treatment of the samples in toluene caused the most effective splitting of the layers among the rest of the solvents. Figure 2a shows colored dispersions of $\mathrm{CF}_{0.08}, \mathrm{CF}_{0.23}$, and $\mathrm{CF}_{0.33}$ in toluene, which were stable after about an hour. The dispersion has a darker color with the decrease in fluorine content in parent fluorinated graphite. Optical absorption spectra measured for the dispersions confirmed the reduction of light transmittance for the low-fluorinated samples (Figure 2b). 

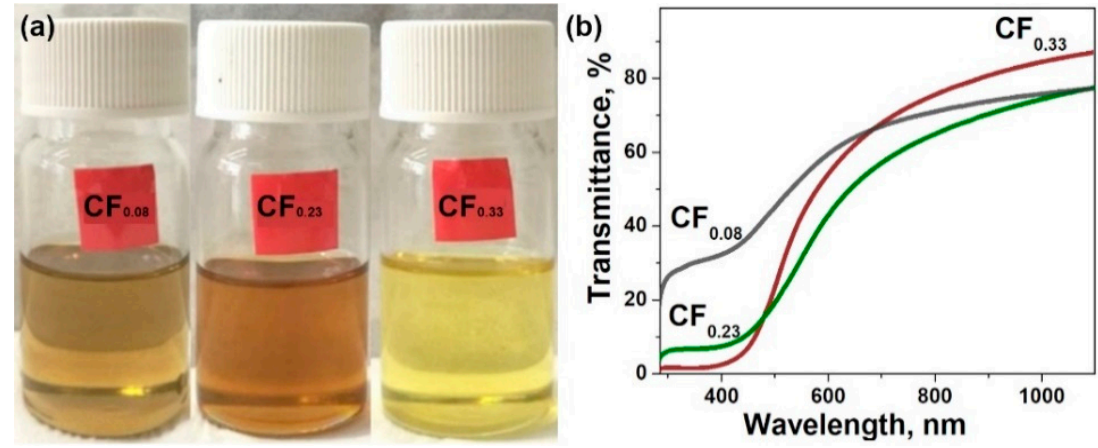

Figure 2. (a) Photographs of fluorinated graphene (FG) dispersions obtained from $\mathrm{CF}_{0.08}, \mathrm{CF}_{0.23}$, and $\mathrm{CF}_{0.33}$ (from left to right) samples; (b) optical absorption spectra in the visible and near-IR regions of FG dispersions in toluene.

Large agglomerates precipitate within an hour of sedimentation of the dispersion, and their removal produces supernatant, which is stable for several hours. To increase the fraction of few-layered FG, additional stages of sonication and centrifugation were used. Optical microscopy showed that this treatment decreases the average size of the FG flakes from 3-20 to 1-5 $\mu \mathrm{m}$ (Figure 3a,b). Moreover, the flakes become more transparent, which undergoes a decrease in the number of adjacent layers. Filtration of FG dispersions after sedimentation of large aggregates and those after additional centrifugation step yielded the films on the CN membranes denoted FG-s and FG-c, respectively. These films can be transferred to various substrates. SEM images of the films deposited from $\mathrm{CF}_{0.08}$ and $\mathrm{CF}_{0.23}$ parents on silicon substrates are compared in Figure $3 c, d$, respectively. Both films consist of wrinkled flakes, however the flakes in less fluorinated material have a flatter structure. Covalent $\mathrm{C}-\mathrm{F}$ bonds destroy the conjugated $\pi$-system of graphene planes, thus reducing their rigidity. Soft layers fold under mechanical treatment in solvent, and the degree of this deformation depends on the number and distribution of C-F bonds. Transparency of an FG film deposited on the PET substrate demonstrates an advantage of the proposed approach to obtain thin graphene-based layers (Figure 3e). The black stripes are electrodes painted by silver paste. Integrity of the film is maintained when the substrate is bent. The thickness of the films obtained was $~ 150-250 \mathrm{~nm}$ as determined by AFM (Figure 3f).
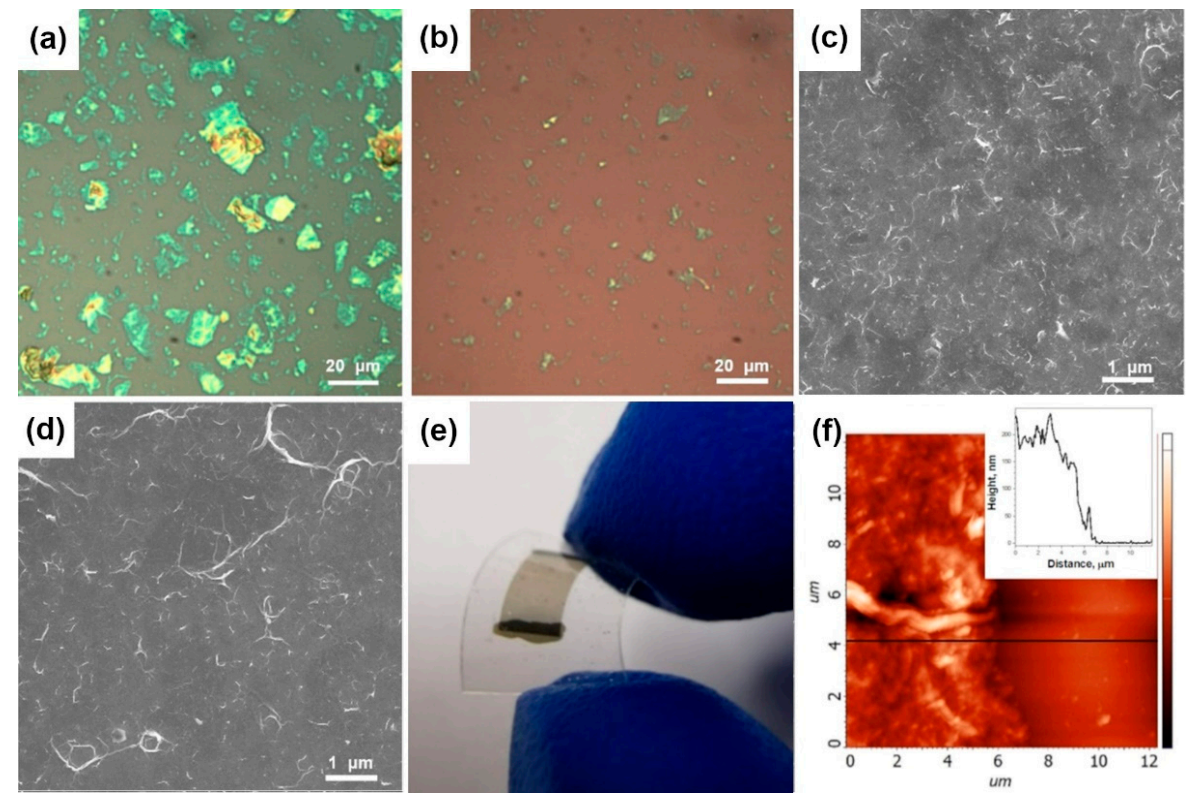

Figure 3. Optical images of FG particles after sedimentation (a) and centrifugation (b) steps. SEM images of FG films obtained from fluorinated graphites $\mathrm{CF}_{0.08}$ (c) and $\mathrm{CF}_{0.23}$ (d). Photo of FG film on PET substrate (e). AFM image of FG film (f), inset shows height profile along black line. 
Raman spectroscopy proved the functionalization of graphene layers in studied materials (Figure 4). Fluorination of graphite led to the appearance of defect-activated peaks D at $1351 \mathrm{~cm}^{-1}$ and $\mathrm{D}^{\prime}$ at $1604 \mathrm{~cm}^{-1}$ (Figure $4 \mathrm{a}$ ), which correspond to the single-phonon intervalley and intravalley scattering processes, respectively. The intensity of these peaks relative to the $G$ peak intensity changes non-monotonically with the fluorine content, which is similar to the previous results on functionalized graphenes [41]. The $\mathrm{D}^{\prime}$ peak is separated from $\mathrm{G}$ peak in the spectrum of $\mathrm{CF}_{0.08}$ powder, and these peaks are merged when the fluorine content increases. All the single-phonon peaks are broadened for the FG films (Figure $4 \mathrm{~b}$ ) as compared to the powder parent materials. The intensity of D peak increases significantly due to the formation of new edge states resulting from the exfoliation process. An increase in the fluorination degree leads to a blue shift of the two-phonon G'-peak for both powders and FG films, which evidences p-type doping of graphene [42].
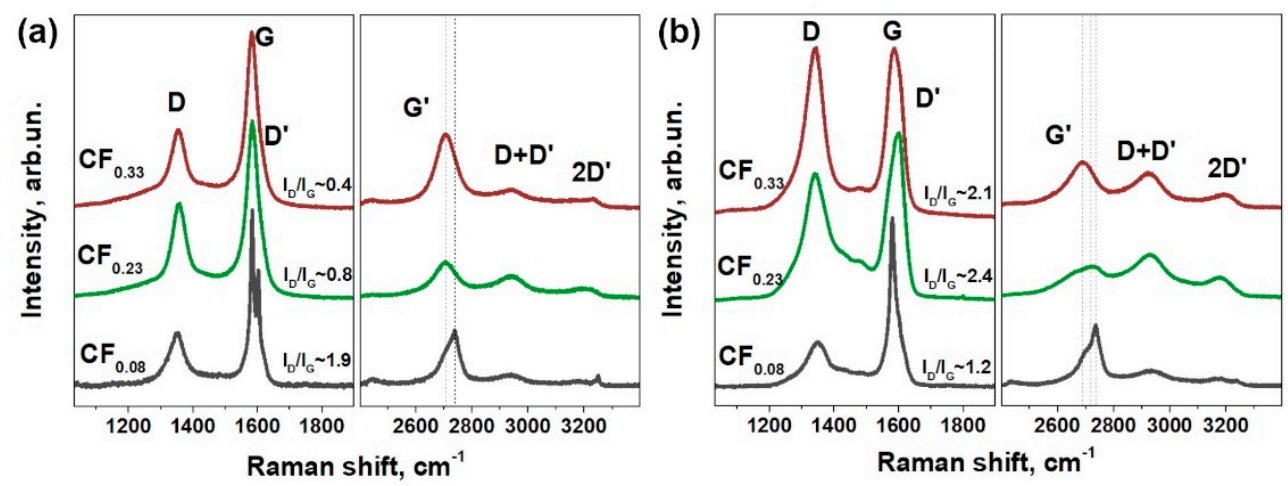

Figure 4. Raman spectra of fluorinated graphite powders (a) and FG films obtained from these powders (b). The spectra were normalized to the intensity of $G$ peak.

\subsection{Sensor Tests}

Effect of the size of FG particles on the sensing performance was examined on the example of FG-s and FG-c films obtained from $\mathrm{CF}_{0.23}$ sample. The tests were performed toward $100 \mathrm{ppm}$ of $\mathrm{NO}_{2}$ in argon. Figure 5a compares dynamic change of the relative current of the films during five cycles of exposure to $\mathrm{NO}_{2}$, followed by purging with pure argon. The FG-s sensor showed ca. $23 \%$ increase in the conductivity in the first cycle. The FG-c film obtained from a smaller fraction of the particles had approximately 1.5-times higher change in the conductivity. The relative response and recovery of the films estimated using Equations (1) and (2) are presented in Figure 5b,c, respectively. Both samples showed a gradual decrease in the response with the cycling (Figure 5b), which could be attributed to the nonreversible adsorption of $\mathrm{NO}_{2}$ at room temperature. The recovery behavior of the sensors was opposite to the response behavior. The FG-s sample showed recovery ca. 30\%, which was higher than that for FG-c (Figure 5c). Similar dependences of the relative response and recovery on the particle size were also observed for the $\mathrm{FG}$ sensors obtained from $\mathrm{CF}_{0.08}$ and $\mathrm{CF}_{0.33}$ samples.

The sensor performance, depending on the fluorine loading, was studied for the thinner FG films obtained after the centrifugation step. Figure $5 \mathrm{~d}$ compares the run-to-run tests of the FG-c films obtained from the fluorinated graphites with different fluorine content. The largest changes in the conductivity under the adsorption of $\mathrm{NO}_{2}$ molecules were detected for the FG-c film obtained from $\mathrm{CF}_{0.33}$ sample. The relative responses of the FG-c sensors in five consecutive operation cycles are presented in Figure 5e. In the first cycle, the response was 21, 28, and $42 \%$ for the sensors prepared from $\mathrm{CF}_{0.08}, \mathrm{CF}_{0.23}$, and $\mathrm{CF}_{0.33}$ samples, respectively. Then, the values decreased, whereas the dependence on the fluorination loading remained. Moreover, the reversibility of the signal at room temperature was strongly improved with the increase in fluorine content in FG film due to better recovery of the sensor (Figure 5f). 


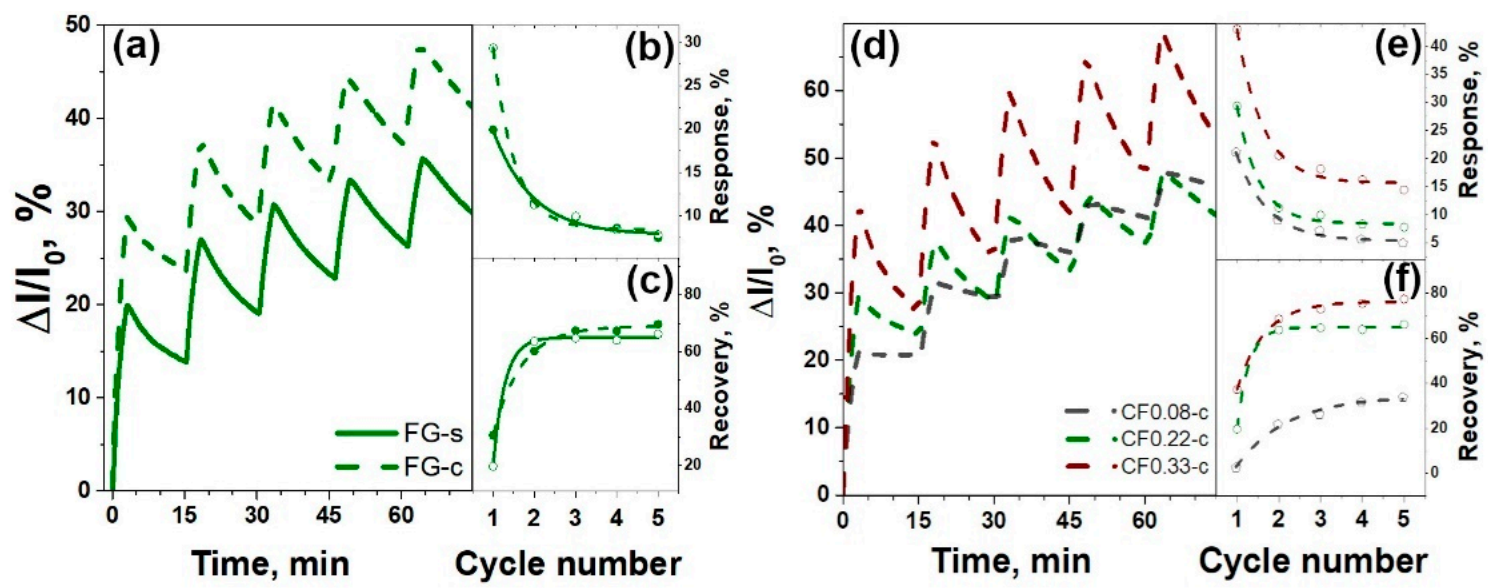

Figure 5. Run-to-run cycling of FG-s and FG-c sensors obtained from graphite fluoride $\mathrm{CF}_{0.23}(\mathbf{a})$ and FG-c sensors obtained from $\mathrm{CF}_{0.08}, \mathrm{CF}_{0.23}$, and $\mathrm{CF}_{0.33}$ samples (d) towards exposure of $100 \mathrm{ppm} \mathrm{NO}_{2}$ at room temperature. Relative response $(\mathbf{b}, \mathbf{e})$ and recovery $(\mathbf{c}, \mathbf{f})$ of the sensors vs. cycle number.

The FG-c films obtained from the fluorinated graphites with the lowest and highest fluorination degree, $\mathrm{CF}_{0.08}$ and $\mathrm{CF}_{0.33}$, were additionally tested at an operation temperature varied from 30 to $80^{\circ} \mathrm{C}$ with a step of $10^{\circ} \mathrm{C}$. At each temperature, one standard cycle was performed to determine the response of the sensor to $100 \mathrm{ppm}$ of $\mathrm{NO}_{2}$ in argon. After the measurement, the sample was heated to $80{ }^{\circ} \mathrm{C}$ and annealed at this temperature for $1 \mathrm{~h}$ to achieve the desorption of $\mathrm{NO}_{2}$, and then it was cooled down to the required operation temperature. Both sensors showed a faster response and recovery with the rise of the operation temperature. The response gradually decreased from 23 to $8.9 \%$ for the FG-c sensor prepared from graphite fluoride $\mathrm{CF}_{0.08}$ and from 33 to $20 \%$ for the FG-c sensor from $\mathrm{CF}_{0.33}$ when the temperature changed from 30 to $80^{\circ} \mathrm{C}$ (Figure 6). The higher fluorinated sample showed much better recovery at all temperatures, which reached a maximum level of ca. $80 \%$ at $80^{\circ} \mathrm{C}$ after 12 min of sensor purging with pure argon.
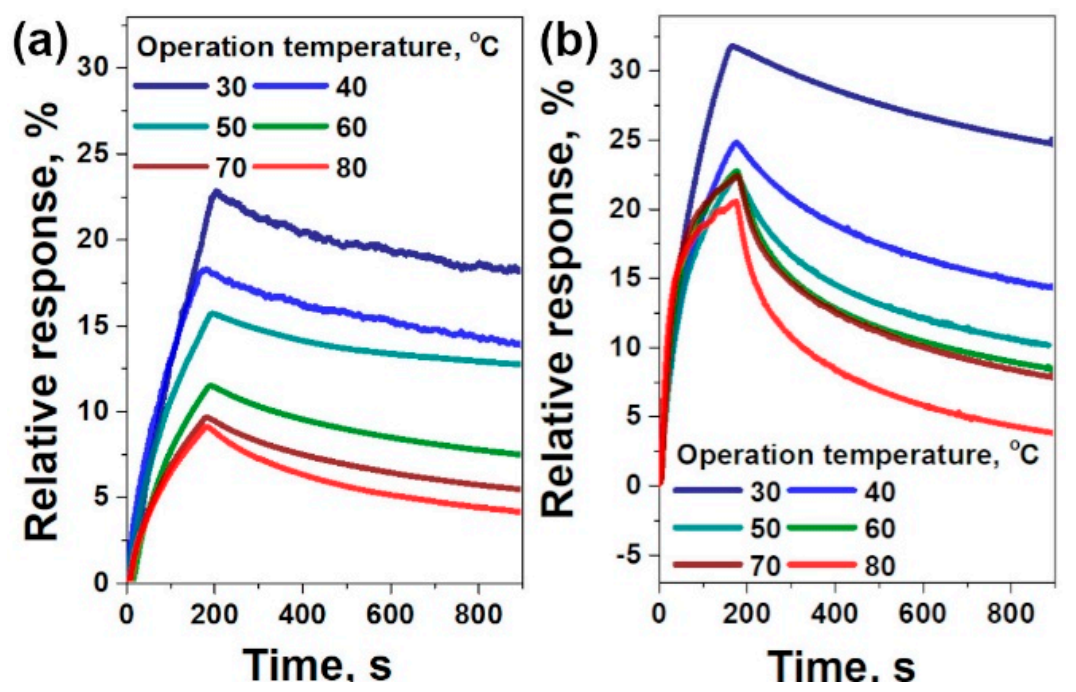

Figure 6. Sensor response of FG-c films prepared from graphite fluorides $\mathrm{CF}_{0.08}$ (a) and $\mathrm{CF}_{0.33}$ (b) towards $100 \mathrm{ppm} \mathrm{NO}_{2}$ at temperatures from 30 to $80{ }^{\circ} \mathrm{C}$.

\section{Discussion}

Since Novoselov and coworkers [1] reported the detection of a single $\mathrm{NO}_{2}$ molecule on mechanically exfoliated graphene, sensor properties of graphene materials as an active material are widely investigated. It was shown that graphene exhibits maximum sensitivity to charge carriers 
corresponded to neutrality point. On the other hand, ideal graphene is chemically inert and does not interact specifically with molecules [43]. The fluctuations in the spectral density of the low-frequency current induced by some gases were proposed as the sensing parameter to enhance selectivity of graphene [44]. Surface functionalization and operation at elevated temperatures reduce the response and recovery times of graphene-based sensors [45]. These sensing parameters can be controlled by the density of the adsorption sites [46]. Fluorination of graphene is especially suitable in that case, because it introduces single-type functional groups at the basal plane and allows tuning the $\mathrm{F} / \mathrm{C}$ ratio. The electrical transport properties of partially fluorinated graphene can be adjusted by tuning the fluorination degree for application in chemical sensors. The charge carrier mobility for single flake devices was found to increase with an increase in the fluorination degree, reaching $2000-3000 \mathrm{~cm}^{2} \mathrm{~V}^{-1} \mathrm{~s}^{-1}$ [47]. In case of thin films, the charge transport is largely affected by edge/edge, edge/plane, and plane/plane junctions. Additionally, we have previously demonstrated that $\mathrm{C}-\mathrm{F}$ groups modify the surface chemistry of graphene forming specific sites for molecule adsorption [31,48]. A similar effect has been achieved for plasma-fluorinated CVD-graphene, whose enhanced sensor performance at room temperature was attributed to p-type doped nature of FG and stronger physical adsorption of ammonia [26]. Park and co-authors have proposed to modify graphene oxide by fluorine and achieved better sensitivity of the fluorinated sensor to ammonia, however all obtained sensors failed to be recovered at room temperature [49].

Covalent attachment of fluorine to the basal plane and the edges of graphene turns part of the carbon atoms from $\mathrm{sp}^{2}$ - to $\mathrm{sp}^{3}$-hybridization state. The XPS study of graphite fluorides used in the present work for the preparation of FG films showed a decrease in the fraction of $\mathrm{sp}^{2}$-hybridized carbon regions and an increase in the population of $\mathrm{sp}^{3}$ defects with the synthesis time and concentration of $\mathrm{BrF}_{3}$ in the reaction mixture (Figure 1). These defects led to reliable p-type doping of the graphene layers and, as a result, to an increase in the resistivity of FG films (Table 1). Such behavior is consistent with previous works on the fluorination [47] and oxidation [50] of graphene. For the FG films obtained using the sedimentation procedure without or with the centrifugation treatment, we observed a decrease in the conductivity by more than three orders of magnitude when the fluorine content in parent fluorinated graphite increased from ca. 7 to ca. 25 at $\%$. The films obtained from the centrifuged dispersions of FG samples showed a lower resistivity for all fluorination degrees due to partial fluorine detachment with an increase in the sonication time. The EDS determined that fluorine content changes from ca. 19 at.\% for graphite powder $\mathrm{CF}_{0.23}$ to ca. 15 at $\%$ for the FG-s film and ca. 14 at $\%$ for the FG-c film.

Table 1. Sample name, fluorine content, and fraction of $\mathrm{sp}^{2}$-hybridized carbon regions in fluorinated graphite powders determined by XPS, thickness $(t)$, sheet resistance $\left(R_{s}\right)$, resistivity $(\rho)$ of the films and their sensor response and recovery.

\begin{tabular}{|c|c|c|c|c|c|c|c|c|}
\hline \multicolumn{2}{|c|}{ Sample } & F Content, at. $\%$ & $\mathrm{C}_{\mathrm{sp2}}$, Fraction \% & $\mathrm{t}, \mathrm{nm}$ & $\mathrm{R}_{\mathrm{s}}, \mathrm{k} \Omega / \square$ & $\rho, \Omega \cdot m$ & Response, \% & Recovery, \% \\
\hline \multirow{2}{*}{$\mathrm{CF}_{0.08}$} & FG-s & \multirow{2}{*}{7} & \multirow{2}{*}{88} & 146 & 66.9 & $9.8 \times 10^{-2}$ & 10 & 21 \\
\hline & FG-c & & & 266 & 75.3 & $2.0 \times 10^{-2}$ & 21 & 2 \\
\hline \multirow{2}{*}{$\mathrm{CF}_{0.23}$} & FG-s & \multirow[b]{2}{*}{19} & \multirow[b]{2}{*}{69} & 210 & 163.9 & $3.4 \times 10^{-2}$ & 15 & 30 \\
\hline & FG-c & & & 210 & 120.9 & $2.5 \times 10^{-2}$ & 29 & 19 \\
\hline $\mathrm{CF}_{0.33}$ & FG-c & 25 & 60 & 215 & 81960 & 17.6 & 43 & 37 \\
\hline
\end{tabular}

Exposure of the FG films to the electron-acceptor $\mathrm{NO}_{2}$ molecule resulted in an increase in the conductivity, which undergoes an increase in the number of charge carriers. We examined the influence of particle size on the sensor response of FG films, obtained from sedimented and centrifuged dispersions of graphite fluoride $\mathrm{CF}_{0.23}$. Run-to-run cycling showed that the small-size fraction has a higher sensor response compared to the large-size fraction (Figure $5 b$ ). This could be attributed to more effective influence of the adsorbed molecule on the charge states of graphene particles due to higher surface-volume ratio [51]. It was shown that monolayer and bilayer graphene have the highest sensitivity to adsorbed molecules [11,52], and the same tendency was observed for carbon 
nanotubes, whereas the sensitivity dropped down from single-walled to multi-walled nanotubes [53]. The recovery of the FG sensors (Figure 5c) showed behavior opposite to the response. As compared to the FG-s sensor, the centrifuged particles had a lower degree of recovery. Probably, small particles formed a more developed pore structure, which trapped the $\mathrm{NO}_{2}$ molecules.

Response and recovery of the FG sensor increased with the fluorine content (Table 1). Fluorinated graphene is a p-type semiconductor, since fluorine is strong electron acceptor [22]. An increase in the content of covalently attached fluorine leads to an increase in the concentration of hole carriers in the material. Additionally, fluorination introduces adsorption sites near the fluorine groups [26,48]. To further highlight the influence of fluorine attachment to the graphene plane on the sensor performance of FG films, we carried out experiments at temperatures between 30 and $80{ }^{\circ} \mathrm{C}$ (Figure 6). An increase in operation temperature resulted in faster saturation of response, which was achieved within ca. $3 \mathrm{~min}$ at $80^{\circ} \mathrm{C}$ for the FG-c sensor prepared from $\mathrm{CF}_{0.33}$. Additionally, we observed retention of the response with an increase in the operation temperature for the sensors. The observed trend could be related to two factors. The first factor is an increase in the concentration of charge carriers in semiconducting materials with temperature [50]. The second factor is the change in conductivity due to an increase in the adsorption rate of molecules on the surface of FG films. We observed the higher impact of the first factor for both sensors, resulting in a decrease in the response ca. 1.5 and ca. three times for the FG-c sensor based on $\mathrm{CF}_{0.33}$ and $\mathrm{CF}_{0.08}$, respectively, with an increase in the operation temperature from 30 to $80^{\circ} \mathrm{C}$. The lower response retention for the former sensor undergoes a higher adsorption rate of $\mathrm{NO}_{2}$ molecules (Figure 6). The higher fluorination degree in that sensor also resulted in better recovery level of $80 \%$ for $12 \mathrm{~min}$ at $80^{\circ} \mathrm{C}$, which is comparable with the recovery of reduced graphene oxide at $150{ }^{\circ} \mathrm{C}$ [54]. This difference could be attributed to lower adsorption energy of $\mathrm{NO}_{2}$ on the fluorinated graphene. Other two-dimensional materials, particularly $\mathrm{MoS}_{2}, \mathrm{WS}_{2}$, and black phosphorus, are widely examined for gas sensing due to their high surface-volume ratio and tunable band gap [55]. Unlike the FG, the fabrication of sensors from the above materials is currently costly. Contaminations and easy oxidation of surfaces require the capping step to prevent problems with reliability and stability of these devices [56].

\section{Conclusions}

Fluorination by a $\mathrm{BrF}_{3} / \mathrm{Br}_{2}$ gaseous mixture at room temperature was used to synthesize fluorinated graphite with a tunable $\mathrm{F} / \mathrm{C}$ ratio by changing the synthesis time and concentration of $\mathrm{BrF}_{3}$. Fluorinated graphites are easily exfoliated in toluene forming stable suspensions when the fluorine content was higher than $7 \%$. The simple imprinting method was developed to prepare uniform FG films with a thickness of $\sim 200 \mathrm{~nm}$ on different substrates. Thin FG films can be transferred on flexible polymer substrates to obtain transparent and bendable films. We revealed the influence of particle fraction and functional composition of FG on the performance of thin-film sensors towards exposure of nitrogen dioxide. The lateral size of FG flakes was changed by additional stages of sonication and centrifugation. It was shown that ultrasonic treatment of fluorinated graphite resulted in partial defluorination of FG flakes for all fluorination degrees. The sensor properties of FG films were tested to $100 \mathrm{ppm} \mathrm{NO} 2$ at room and elevated temperatures. It was shown that the particle fraction affects the relative response via efficiency of charge transfer between the molecule and graphene layers. The sensor characteristics of FG enhanced with the fluorination loading. The fluorine groups act as a scattering center, dopant, and adsorption site, which improves electrical response and response/recovery rates. Regarding that, we stated a high efficiency of fluorinated graphites for the fabrication of thin-film graphene-based sensors due to their excellent exfoliation in organic solvents and tunable electronic properties.

Author Contributions: Writing, measurements, and characterization, V.I.S.; measurements and characterization, M.O.B.; synthesis, G.N.C. and D.V.P.; measurements, P.N.G. and I.P.A.; writing, L.G.B.; data analysis and methodology, A.V.O. All authors have read and agreed to the published version of the manuscript.

Funding: The work of the materials synthesis was funded by the Russian Foundation for Basic Research (Grant No. 18-29-19073). 
Acknowledgments: We are grateful to Boris A. Kolesov for the Raman spectra.

Conflicts of Interest: The authors declare no conflict of interest.

\section{References}

1. Schedin, F.; Geim, A.K.; Morozov, S.V.; Hill, E.H.; Blake, P.; Katsnelson, M.I.; Novoselov, K.S. Detection of individual gas molecules adsorbed on graphene. Nat. Mater. 2007, 6, 652-655. [CrossRef] [PubMed]

2. Hu, N.; Yang, Z.; Wang, Y.; Zhang, L.; Wang, Y.; Huang, X.; Wei, H.; Wei, L.; Zhang, Y. Ultrafast and sensitive room temperature $\mathrm{NH}_{3}$ gas sensors based on chemically reduced graphene oxide. Nanotechnology 2013, 25, 25502. [CrossRef] [PubMed]

3. Fattah, A.; Khatami, S.M.A. Selective $\mathrm{H}_{2} \mathrm{~S}$ gas sensing with a graphene/n-Si schottky diode. IEEE Sens. J. 2014, 14, 4104-4108. [CrossRef]

4. Yoon, H.J.; Jun, D.H.; Yang, J.H.; Zhou, Z.; Yang, S.S.; Cheng, M.M.-C. Carbon dioxide gas sensor using a graphene sheet. Sens. Actuators B Chem. 2011, 157, 310-313. [CrossRef]

5. Jeong, H.Y.; Lee, D.-S.; Choi, H.K.; Lee, D.H.; Kim, J.-E.; Lee, J.Y.; Lee, W.J.; Kim, S.O.; Choi, S. Flexible room-temperature $\mathrm{NO}_{2}$ gas sensors based on carbon nanotubes/reduced graphene hybrid films. Appl. Phys. Lett. 2010, 96, 213105. [CrossRef]

6. Yuan, W.; Liu, A.; Huang, L.; Li, C.; Shi, G. High-performance $\mathrm{NO}_{2}$ sensors based on chemically modified graphene. Adv. Mater. 2012, 25, 766-771. [CrossRef]

7. Fowler, J.D.; Allen, M.J.; Tung, V.C.; Yang, Y.; Kaner, R.B.; Weiller, B.H. Practical chemical sensors from chemically derived graphene. ACS Nano 2009, 3, 301-306. [CrossRef]

8. Moos, R.; Müller, R.; Plog, C.; Knezevic, A.; Leye, H.; Irion, E.; Braun, T.; Marquardt, K.-J.; Binder, K. Selective ammonia exhaust gas sensor for automotive applications. Sens. Actuators B Chem. 2002, 83, 181-189. [CrossRef]

9. Jiang, Q.; Kresin, F.; Bregt, A.K.; Kooistra, L.; Pareschi, E.; Van Putten, E.; Volten, H.; Wesseling, J. Citizen sensing for improved urban environmental monitoring. J. Sens. 2016, 2016, 1-9. [CrossRef]

10. Robinson, J.T.; Perkins, F.K.; Snow, E.S.; Wei, Z.; Sheehan, P.E. Reduced graphene oxide molecular sensors. Nano Lett. 2008, 8, 3137-3140. [CrossRef]

11. Seekaew, Y.; Phokharatkul, D.; Wisitsoraat, A.; Wongchoosuk, C. Highly sensitive and selective room-temperature $\mathrm{NO}_{2}$ gas sensor based on bilayer transferred chemical vapor deposited graphene. Appl. Surf. Sci. 2017, 404, 357-363. [CrossRef]

12. Niu, F.; Tao, L.-M.; Deng, Y.-C.; Wang, Q.-H.; Song, W. Phosphorus doped graphene nanosheets for room temperature $\mathrm{NH}_{3}$ sensing. New J. Chem. 2014, 38, 2269. [CrossRef]

13. Lv, R.; Chen, G.; Li, Q.; McCreary, A.; Botello-Méndez, A.R.; Morozov, S.V.; Liang, L.; Declerck, X.; Perea-López, N.; Cullen, D.A.; et al. Ultrasensitive gas detection of large-area boron-doped graphene. Proc. Natl. Acad. Sci. USA 2015, 112, 14527-14532. [CrossRef] [PubMed]

14. Choi, Y.R.; Yoon, Y.-G.; Choi, K.; Kang, J.H.; Shim, Y.-S.; Kim, Y.H.; Chang, H.J.; Lee, J.-H.; Park, C.R.; Kim, S.Y.; et al. Role of oxygen functional groups in graphene oxide for reversible room-temperature $\mathrm{NO}_{2}$ sensing. Carbon 2015, 91, 178-187. [CrossRef]

15. Sreeprasad, T.S.; Berry, V. How do the electrical properties of graphene change with its functionalization? Small 2012, 9, 341-350. [CrossRef]

16. Chen, N.; Huang, X.; Qu, L. Heteroatom substituted and decorated graphene: Preparation and applications. Phys. Chem. Chem. Phys. 2015, 17, 32077-32098. [CrossRef]

17. Sturala, J.; Luxa, J.; Pumera, M.; Sofer, Z. Chemistry of graphene derivatives: Synthesis, applications, and perspectives. Chem. Eur. J. 2018, 24, 5992-6006. [CrossRef]

18. Bouša, D.; Luxa, J.; Mazánek, V.; Jankovský, O.; Sedmidubský, D.; Klímová, K.; Pumera, M.; Sofer, Z. Toward graphene chloride: Chlorination of graphene and graphene oxide. RSC Adv. 2016, 6, 66884-66892. [CrossRef]

19. Jankovský, O.; Lojka, M.; Luxa, J.; Sedmidubský, D.; Tomanec, O.; Zbořil, R.; Pumera, M.; Sofer, Z. Selective bromination of graphene oxide by the hunsdiecker reaction. Chem. A Eur. J. 2017, 23, 10473-10479. [CrossRef]

20. Mazánek, V.; Jankovský, O.; Luxa, J.; Sedmidubský, D.; Janoušek, Z.; Šembera, F.; Mikulics, M.; Sofer, Z. Tuning of fluorine content in graphene: Towards large-scale production of stoichiometric fluorographene. Nanoscale 2015, 7, 13646-13655. [CrossRef] 
21. Bulusheva, L.G.; Okotrub, A.V. Electronic Structure of Fluorinated Graphene In New Fluorinated Carbons: Fundamentals and Applications; Boltalina, O.V., Nakajima, T., Eds.; Elsevier Inc.: Amsterdam, The Netherlands, 2017; pp. 177-213.

22. Feng, W.; Long, P.; Feng, Y.; Li, Y. Two-dimensional fluorinated graphene: Synthesis, Structures, properties and applications. Adv. Sci. 2016, 3, 1500413. [CrossRef] [PubMed]

23. Wang, Z.; Wang, J.; Li, Z.; Gong, P.; Liu, X.; Zhang, L.; Ren, J.; Wang, H.; Yang, S. Synthesis of fluorinated graphene with tunable degree of fluorination. Carbon 2012, 50, 5403-5410. [CrossRef]

24. Nebogatikova, N.A.; Antonova, I.; Prinz, V.; Kurkina, I.I.; Vdovin, V.I.; Aleksandrov, G.N.; Timofeev, V.B.; Smagulova, S.A.; Zakirov, E.R.; Kesler, V.G. Fluorinated graphene dielectric films obtained from functionalized graphene suspension: Preparation and properties. Phys. Chem. Chem. Phys. 2015, 17, 13257-13266. [CrossRef] [PubMed]

25. Lee, W.H.; Suk, J.W.; Chou, H.; Lee, J.; Hao, Y.; Wu, Y.; Piner, R.; Akinwande, D.; Kim, H.R.; Ruoff, R.S. Selective-area fluorination of graphene with fluoropolymer and laser irradiation. Nano Lett. 2012, 12, 2374-2378. [CrossRef]

26. Zhang, H.; Fan, L.; Dong, H.; Zhang, P.; Nie, K.; Zhong, J.; Li, Y.; Guo, J.; Sun, X. Spectroscopic investigation of plasma-fluorinated monolayer graphene and application for gas sensing. ACS Appl. Mater. Interfaces 2016, 8, 8652-8661. [CrossRef]

27. Nair, R.R.; Ren, W.; Jalil, R.; Riaz, I.; Kravets, V.G.; Britnell, L.; Blake, P.; Schedin, F.; Mayorov, A.S.; Yuan, S.; et al. Fluorographene: A two-dimensional counterpart of teflon. Small 2010, 6, 2877-2884. [CrossRef]

28. Bulusheva, L.G.; Fedoseeva, Y.; Flahaut, E.; Rio, J.; Ewels, C.; Koroteev, V.O.; Van Lier, G.; Vyalikh, D.V.; Okotrub, A.V. Effect of the fluorination technique on the surface-fluorination patterning of double-walled carbon nanotubes. Beilstein J. Nanotechnol. 2017, 8, 1688-1698. [CrossRef]

29. Chekhova, G.; Pinakov, D.; Shubin, Y.; Fadeeva, V.; Tikhova, V.; Okotrub, A.; Bulusheva, L. Room temperature synthesis of fluorinated graphite intercalation compounds with low fluorine loading of host matrix. J. Fluor. Chem. 2020, 232, 109482. [CrossRef]

30. Vyalikh, A.; Bulusheva, L.; Chekhova, G.N.; Pinakov, D.; Okotrub, A.V.; Scheler, U. Fluorine patterning in room-temperature fluorinated graphite determined by solid-state NMR and DFT. J. Phys. Chem. C 2013, 117, 7940-7948. [CrossRef]

31. Katkov, M.V.; Sysoev, V.I.; Gusel'Nikov, A.V.; Asanov, I.P.; Bulusheva, L.G.; Okotrub, A.V. A backside fluorine-functionalized graphene layer for ammonia detection. Phys. Chem. Chem. Phys. 2015, 17, 444-450. [CrossRef]

32. Okotrub, A.V.; Asanov, I.P.; Yudanov, N.F.; Babin, K.S.; Gusel'Nikov, A.V.; Nedoseikina, T.I.; Gevko, P.N.; Bulusheva, L.G.; Osváth, Z.; Biró, L.P. Development of graphene layers by reduction of graphite fluoride $\mathrm{C}_{2} \mathrm{~F}$ surface. Phys. Status Solidi B 2009, 246, 2545-2548. [CrossRef]

33. Okotrub, A.V.; Yudanov, N.F.; Asanov, I.P.; Vyalikh, D.V.; Bulusheva, L. Anisotropy of chemical bonding in semifluorinated graphite $\mathrm{C}_{2} \mathrm{~F}$ revealed with angle-resolved X-ray absorption spectroscopy. ACS Nano 2012, 7, 65-74. [CrossRef] [PubMed]

34. Asanov, I.; Bulusheva, L.; Dubois, M.; Yudanov, N.; Alexeev, A.; Makarova, T.; Okotrub, A.V. Graphene nanochains and nanoislands in the layers of room-temperature fluorinated graphite. Carbon 2013, 59, 518-529. [CrossRef]

35. Tressaud, A.; Durand, E.; Labrugere, C. Surface modification of several carbon-based materials: Comparison between $\mathrm{CF}_{4} \mathrm{rf}$ plasma and direct $\mathrm{F}_{2}$-gas fluorination routes. J. Fluor. Chem. 2004, 125, 1639-1648. [CrossRef]

36. Wang, B.; Wang, J.; Zhu, J. Fluorination of graphene: A spectroscopic and microscopic study. ACS Nano 2014, 8, 1862-1870. [CrossRef]

37. Fedoseeva, Y.; Bulusheva, L.G.; Okotrub, A.V.; Vyalikh, D.V.; Fonseca, A. A comparative study of argon ion irradiated pristine and fluorinated single-wall carbon nanotubes. J. Chem. Phys. 2010, 133, 224706. [CrossRef]

38. Bulusheva, L.; Tur, V.; Fedorovskaya, E.; Asanov, I.; Pontiroli, D.; Riccò, M.; Okotrub, A.V. Structure and supercapacitor performance of graphene materials obtained from brominated and fluorinated graphites. Carbon 2014, 78, 137-146. [CrossRef]

39. Gong, P.; Wang, Z.; Wang, J.; Wang, H.; Li, Z.; Fan, Z.; Xu, Y.; Han, X.; Yang, S. One-pot sonochemical preparation of fluorographene and selective tuning of its fluorine coverage. J. Mater. Chem. 2012, 22, 16950. [CrossRef] 
40. Zhu, M.; Xie, X.; Guo, Y.; Chen, P.; Ou, X.; Yu, G.; Liu, M. Fluorographene nanosheets with broad solvent dispersibility and their applications as a modified layer in organic field-effect transistors. Phys. Chem. Chem. Phys. 2013, 15, 20992. [CrossRef]

41. Eckmann, A.; Felten, A.; Verzhbitskiy, I.A.; Davey, R.; Casiraghi, C. Raman study on defective graphene: Effect of the excitation energy, type, and amount of defects. Phys. Rev. B 2013, 88, 1-11. [CrossRef]

42. Wu, J.; Xie, L. Structural quantification for graphene and related two-dimensional materials by Raman spectroscopy. Anal. Chem. 2018, 91, 468-481. [CrossRef]

43. Wehling, T.O.; Novoselov, K.S.; Morozov, S.V.; Vdovin, E.E.; Katsnelson, M.I.; Geim, A.K.; Lichtenstein, A.I. Molecular doping of graphene. Nano Lett. 2008, 8, 173-177. [CrossRef] [PubMed]

44. Rumyantsev, S.L.; Liu, G.; Shur, M.; Potyrailo, R.A.; Balandin, A.A. Selective gas sensing with a single pristine graphene transistor. Nano Lett. 2012, 12, 2294-2298. [CrossRef] [PubMed]

45. Rumyantsev, S.L.; Liu, G.; Potyrailo, R.A.; Balandin, A.A.; Shur, M. Selective sensing of individual gases using graphene devices. IEEE Sens. J. 2013, 13, 2818-2822. [CrossRef]

46. Robinson, J.A.; Snow, E.S.; Bădescu Ştefan, C.; Reinecke, T.L.; Perkins, F.K. Role of defects in single-walled carbon nanotube chemical sensors. Nano Lett. 2006, 6, 1747-1751. [CrossRef] [PubMed]

47. Koleśnik-Gray, M.; Sysoev, V.I.; Gollwitzer, S.; Pinakov, D.V.; Chekhova, G.N.; Bulusheva, L.G.; Okotrub, A.V.; Krstić, V. Electrical transport in devices based on edge-fluorinated graphene. Adv. Electron. Mater. 2018, 4, 1-5. [CrossRef]

48. Sysoev, V.I.; Okotrub, A.V.; Asanov, I.P.; Gevko, P.N.; Bulusheva, L. Advantage of graphene fluorination instead of oxygenation for restorable adsorption of gaseous ammonia and nitrogen dioxide. Carbon 2017, 118, 225-232. [CrossRef]

49. Park, M.-S.; Kim, K.H.; Kim, M.-J.; Lee, Y.-S. $\mathrm{NH}_{3}$ gas sensing properties of a gas sensor based on fluorinated graphene oxide. Colloids Surf. A Physicochem. Eng. Asp. 2016, 490, 104-109. [CrossRef]

50. Eda, G.; Chhowalla, M. Graphene-based composite thin films for electronics. Nano Lett. 2009, 9, 814-818. [CrossRef]

51. Sysoev, V.I.; Bulusheva, L.G.; Asanov, I.P.; Shubin, Y.V.; Okotrub, A.V. Thermally exfoliated fluorinated graphite for $\mathrm{NO}_{2}$ gas sensing. Phys. Status Solidi B 2016, 253, 2492-2498. [CrossRef]

52. Chen, G.; Paronyan, T.M.; Harutyunyan, A.R. Sub-ppt gas detection with pristine graphene. Appl. Phys. Lett. 2012, 101, 53119. [CrossRef]

53. Han, T.; Nag, A.; Mukhopadhyay, S.; Xu, Y. Carbon nanotubes and its gas-sensing applications: A review. Sens. Actuators A Phys. 2019, 291, 107-143. [CrossRef]

54. Prezioso, S.; Perrozzi, F.; Giancaterini, L.; Cantalini, C.; Treossi, E.; Palermo, V.; Nardone, M.; Santucci, S.; Ottaviano, L. Graphene oxide as a practical solution to high sensitivity gas sensing. J. Phys. Chem. C 2013, 117, 10683-10690. [CrossRef]

55. Donarelli, M.; Ottaviano, L. 2D Materials for gas sensing applications: A review on graphene oxide, $\mathrm{MoS}_{2}$, $\mathrm{WS}_{2}$ and phosphorene. Sensors 2018, 18, 3638. [CrossRef] [PubMed]

56. Liu, G.; Rumyantsev, S.L.; Jiang, C.; Shur, M.; Balandin, A.A. Selective gas sensing with h-BN capped $\mathrm{MoS}_{2}$ heterostructure thin-film transistors. IEEE Electron Device Lett. 2015, 36, 1202-1204. [CrossRef] 